\title{
COMMENTARY
}

\section{Pharmacists Are Not Mid-Level Providers}

Gina D. Moore, PharmD, MBA, ${ }^{a}$ Lynette R. Bradley-Baker, PhD,${ }^{b}$ Nidhi Gandhi, PharmD,${ }^{b}$ Diane B. Ginsburg, PhD, MS, ${ }^{c}$ Karl Hess, PharmD, ${ }^{\text {dee }}$ Clark Kebodeaux, PharmD, ${ }^{\mathrm{f}}$ Jody L. Lounsbery, PharmD, ${ }^{\mathrm{g}}$ Lisa M. Meny, PharmD, ${ }^{\text {h }}$ Elizabeth K. Tanner, PhD, MS, ${ }^{\mathrm{i}}$ Anne Lin, PharmD ${ }^{\mathrm{b}, \mathrm{j}}$

${ }^{\text {a }}$ University of Colorado, Skaggs School of Pharmacy and Pharmaceutical Science, Aurora, Colorado

${ }^{\mathrm{b}}$ American Association of Colleges of Pharmacy, Arlington, Virginia

${ }^{\mathrm{c}}$ The University of Texas at Austin, College of Pharmacy, Austin, Texas

${ }^{\mathrm{d}}$ Chapman University, School of Pharmacy, Irvine, California

${ }^{\mathrm{e}}$ Editorial Board Member, American Journal of Pharmaceutical Education, Arlington, Virginia

${ }^{\mathrm{f}}$ University of Kentucky, School of Pharmacy, Lexington, Kentucky

${ }^{\mathrm{g}}$ University of Minnesota, College of Pharmacy, Minnesota, Minnesota

${ }^{\mathrm{h}}$ Ferris State University, College of Pharmacy, Grand Rapids, Michigan

${ }^{\mathrm{i}}$ Johns Hopkins University, School of Nursing, Baltimore, Maryland

${ }^{\mathrm{j}}$ Notre Dame of Maryland, School of Pharmacy, Baltimore, Maryland

Corresponding Author: Lynette R. Bradley-Baker, American Association of Colleges of Pharmacy, 1400 Crystal Dr, Suite 300, Arlington, VA 22202. Tel: 703-479-3799. Email: 1bbaker@ aacp.org

Submitted January 25, 2021; accepted June 20, 2021; ePublished July 2021

Written by members of the 2021 AACP Professional Affairs Committee, this commentary describes the perspective on why pharmacists should not be classified as "mid-level" providers. Beyond the connotation that this classification implies that there are different levels or a hierarchy of providers, it is important to recognize that all health care providers bring unique and essential knowledge and contributions to the health care team and to patients. Timely issues germane to pharmacists, including dependent and independent practice, provider status, and professional identity also contribute to the rationale that pharmacists, just like all other health care providers, should be classified by their professional identity. While use of the term "mid-level" provider to identify various practitioners may not seem consequential in today's health care environment, it is important to recognize that words do matter in attributing value, and the contributions of all health care providers should be recognized as equally important to the patient care team.

Keywords: collaborative drug therapy management, pharmacists, professional identity

It is interesting how the use of a phrase or classification can lead to a deeper conversation. This occurred as the 2021 American Association of Colleges of Pharmacy (AACP) Professional Affairs Committee (PAC) was working on its charges when the question was raised why pharmacists are referenced as other "mid-level" providers. It was not the intention of AACP President Lin to create controversy by including this term in the committee charge-in fact, it is one that has been used to classify pharmacists. ${ }^{1,2}$

The World Health Organization defines mid-level health providers as individuals trained at a higher education institute for at least 2-3 years who are authorized to work autonomously to diagnose, manage and treat illness. ${ }^{3}$ Pharmacists are also classified as mid-level providers by the Drug Enforcement Administration (DEA), in reference to their ability to dispense controlled substances. ${ }^{2}$ However, the term "mid-level" does not capture the specialized knowledge and comprehensive skills that pharmacists possess and utilize in providing patient-centered care, nor does it adequately reflect the advanced education and training that pharmacists receive or the fact that many graduates go on to complete post-graduate training. The PAC does not believe nor claim that pharmacists are superior to other health care providers, but does believe that a title which implies a rank in order is not appropriate. Each profession should be recognized for the expertise it brings to patient care and to the health care team. Other health professions who are also classified as "midlevel" have expressed their opposition this term as well. ${ }^{4,5}$ During the PAC discussion of the term "mid-level", additional substantive issues evolved, including the unique contributions of pharmacists to patients and the health care team, provider status, professional identity formation, and public perception of pharmacists. These issues are of paramount importance as pharmacists continue to demonstrate their unique role and level of accountability as a provider on the health care team and to patients. 
Pharmacists are medication experts with the unique ability to optimize the medication-related outcomes of patients. When pharmacists practice at the fullest extent of the professions' scope of practice or "at the top of their education," they participate in a broad spectrum of services, such as managing chronic disease and medication management, conducting health and wellness visits, and providing preventative care services. ${ }^{6}$ While it is recognized that the scope of practice of health care providers can vary significantly, some providers are delegated to manage less complicated and complex patients. This is not true for pharmacists. Pharmacists are best utilized when managing the medications of complex patients with multiple medications and co-morbid disease states. Within an interprofessional team of health care providers, pharmacists play a vital role as experts in managing the pharmacotherapy of patient care to improve health and quality of life. ${ }^{7}$

Collaborative Drug Therapy Management (CDTM) is the most common way pharmacists can prescribe medications under an established protocol. Forty-nine states and the District of Columbia allow pharmacists to enter into CDTM agreements which may include initiation or modification of therapy, ordering of laboratory tests, and medication administration. ${ }^{8}$ Agreements or protocols vary by state with some states requiring additional credentials (eg, certification) to participate in CDTM agreements. These practices are dependent on varying levels of oversight by the physician. State statutes and regulations establish requirements for CDTM agreements between pharmacists and physicians. Although this a dependent relationship, the pharmacist and supervising physician are both legally responsible for the actions delineated in the agreement. Ultimately the physician is responsible for the outcome; however, the pharmacist has a legal duty which can be breached for negative outcomes and/or failure to follow the protocol.

There are also practices and patient care activities that allow pharmacists to function as independent health care providers, such as those mandated in statewide protocols; however, these are limited. ${ }^{8}$ As state practice acts change, the potential for an increase in independent pharmacist practices exists; however, like provider status, the profession has a long journey before pharmacists can be considered independent health care providers in all settings.

Pharmacists obtaining provider status in the Medicare Part B program would provide a recognized reimbursement mechanism and would also increase beneficiary access to advanced patient-care services. Pharmacist-provided services, such as comprehensive medication management, have been shown to improve patient outcomes and reduce overall health care costs. ${ }^{9}$ Provider status will also assist pharmacists in providing necessary patient care due to the looming shortage of primary care providers that is predicted to occur in the next decade. ${ }^{10}$

The COVID-19 pandemic has placed an enormous strain on the US health care system causing a surge in hospitalization utilization while also having the effect of discouraging others from accessing routine care. Through authorizations by the US Department of Health and Human Services (HHS), pharmacists are now able to administer COVID tests and vaccinations, as well as routine vaccines to qualified individuals. ${ }^{11}$ By preempting existing state laws, these HHS authorizations have essentially allowed pharmacists to practice at the top of their education to provide critical patient care services during this great time of need. During this time, pharmacists can and have been performing patient assessments in order to help triage patients to the appropriate level of care, recommending self-care options when appropriate, and providing needed drug information to other health care practitioners and patients. ${ }^{12}$ These newly authorized pharmacist roles should remain and in fact expand when the COVID-19 pandemic ends to further validate pharmacists for their unique contributions as essential health care providers within the health care system.

Referring to pharmacists as mid-level providers contributes to the lack of clarity about their role and hampers professional identity formation for student pharmacists and pharmacists. Professional identity formation is critical to the development of pharmacists and their understanding of the role that they play on the health care team. In medicine, professional identity has been defined as "a representation of self, achieved in stages over time during which the characteristics, values, and norms of the medical profession are internalized, resulting in an individual thinking, acting, and feeling like a physician."13 Pharmacy as a profession has struggled with forming its unique professional identity. Multiple identities have been described, with the list of roles growing over time without the desired result. ${ }^{14,15}$ These roles and their evolving nomenclatures may have led to potential confusion and both an internal and external under appreciation of the uniqueness of pharmacists contributions to health care.

All pharmacists must think, act and feel like pharmacists. Gregory and Austin described a "fused personalprofessional identity," in which pharmacists not only practice pharmacy but are pharmacists existentially. ${ }^{16}$ In order to be appreciated as health care providers, all pharmacists must take responsibility for and ownership of their roles. Those pharmacists in academia must be cognizant of their own professional identity to educate future pharmacists. Without attention to professional identity formation, student pharmacists may struggle to form their own professional identities. ${ }^{17}$ The education and training provided to student pharmacists contributes to their ability to grow and think like pharmacists practicing at the top of their license. The 2020 AACP Student Affairs Standing Committee actively worked on professional identity formation including proposing the following adopted AACP policy statement: AACP encourages colleges and schools of pharmacy to advance education that is aimed at the intentional formation of professional identity 
(ie, thinking, feeling and acting like a pharmacist) and developed and implemented in cooperation with professional pharmacy organizations within the broader pharmacy profession. ${ }^{18}$

Regardless of titles used in interprofessional settings, perspectives of the pharmacy profession are often shaped by patients and the public at-large. While many practitioners are aware of the knowledge and skill set associated with a Doctor of Pharmacy degree, it may not be as clear to the public. For example, the Bureau of Labor Statistics separates 'Pharmacists' from 'Health care diagnosing or treating practitioners' as a category of employment, predicting a 10\% growth for the latter and a $3 \%$ decrease for the former through 2029. ${ }^{19}$ While pharmacists are held in high regard in public trust, the public perception of their role is not one of a mid-level provider. ${ }^{20}$

The pharmacy profession will continue to move forward to positively impact patient care. We need to continue to communicate and demonstrate the added value pharmacists provide to patients, to caregivers, and to the health care team. We must continue to advocate for the expansion of the role of pharmacists in laws and regulations at the national and state level. We need to consciously expand our communication about who pharmacists are and what pharmacists do to those outside of the profession - to those who are not aware; to those who are being educated with future pharmacists; to those in other health care professions. With the requirement of interprofessional education for all health professions, the time is now to educate our colleagues in other professions about the pharmacist role and scope of practice. Our efforts for greater awareness and recognition of pharmacists' expertise will be greatly assisted when there are more diverse voices advocating and asking for services that can only be provided by pharmacists.

While the roles on patient care teams can sometimes be shared and overlapping, each health care provider does bring a unique role to a team and therefore should not be referenced by the term "mid-level." We, as academicians and pharmacists, should also be cognizant not to use such terms to describe any health care provider. Pharmacists must continue to have the necessary, yet challenging conversations internally and external to the profession regarding who they are--developing, defining and communicating their professional identity is more important now than ever before. Pharmacists, like all health care providers, are not mid-level--but rather are equally committed to optimizing the health and care of patients. That is a classification that everyone on the health care team can be proud of.

\section{REFERENCES}

1. Ross LA. Pharmacists as mid-level practitioners/providers. Ann Pharmacother. 2011;45(6):810-812. doi:150.1345/aph.1Q133.

2. US Department of Justice. Drug Enforcement Administration. Mid-Level Practitioners Authorization by State. https://www.deadiversion.usdoj.gov/drugreg/practioners/mlp_by_state.pdf. Accessed June 17, 2021.

3. World Health Organization: Mid-level providers-a promising resources to achieve the health Millennium Development Goals. Final_MLP_web_2.pdf (who.int). Accessed June 17, 2021.

4. Anderson J: The Next Person Who Calls Me a Mid-Level Provider. https://www.clinicaladvisor.com/home/thewaiting-room/the-next-person-who-calls-me-a-mid-level-provider/. Accessed June 17, 2021.

5. American Association of Nurse Practitioners: Use of Terms Such as Mid-level Provider and Physician Extender. https://www.aanp.org/advocacy/advocacy-resource/position-statements/use-of-terms-such-as-mid-level-providerand-physician-extender. Accessed June 17, 2021.

6. American Pharmacists Association Action Center: Pharmacists Provide Care. https://actioncenter.pharmacist.com/campaign/sign-up-pharmacists-provide-care/. Accessed June 17, 2021.

7. World Medical Association. WMA statement on the relationship between physicians and pharmacists in medicinal therapy. https://www.wma.net/policies-post/wma-statement-on-the-relationship-between-physiciansand-pharmacists-in-medicinal-therapy/. Accessed June 17, 2021.

8. NASPA. Pharmacist Prescribing: Statewide Protocols and More. https://naspa.us/resource/swp/\#: :text=The\%20term\%20statewide\%20protocol\%20refers, when\%20providing\%20 a\%20clinical\%20service. Accessed June 17, 2021.

9. Manolakis PG, Skelton JB. Pharmacists' contributions to primary care in the United States collaborating to address unmet patient care needs: The merging role of pharmacists to address the shortage of primary care providers. Am J Pharm Educ. 2010; 74(10): aj7410S7,

10. NASPA. United States Pharmacists: Improving People's Health. https://naspa.us/resource/2020-state-fact-sheets/. Accessed June 17, 2021.

11. US Department of Health and Human Services. PREP Act Authorization for Pharmacies Distributing and Administering Certain Covered Countermeasures. https://www.hhs.gov/guidance/sites/default/files/hhs-guidancedocuments/prep-act-authorization-pharmacies-administering-covered-countermeasures.pdf. Accessed June 17, 2021. 
12. Hess K, Bach A, Won K, et al. Community Pharmacists Roles During the COVID-19 Pandemic. J Pharm Pract. 2020 Dec 15:897190020980626. doi: 10.1177/0897190020980626. Epub ahead of print. PMID: 33317371.

13. Cruess RL, Cruess SR, Boudreau JD, et al. Reframing medical education to support professional identity formation. Acad Med. 2014;89(11):1446-1451. doi:10.1097/ACM.0000000000000427.

14. Elvey R, Hassell K, Hall J. Who do you think you are? Pharmacists' perceptions of their professional identity. Int J Pharm Pract. 2013;21(5):322-332. doi:10.1111/ijpp.12019.

15. Kellar J, Paradis E, van der Vleuten CPM, et al. A Historical Discourse Analysis of Pharmacist Identity in Pharmacy Education. Am J Pharm Educ. 2020;84(9). doi:10.5688/ajpe7864.

16. Gregory P, Austin Z. Pharmacists' lack of profession-hood: Professional identity formation and its implications for practice. Can Pharm J (Ott). 2019;152(4):251-256. doi:10.1177/1715163519846534.

17. Noble C, McKauge L, Clavarino A. Pharmacy student professional identity formation: a scoping review. Integr Pharm Res Pract. 2019;8:15-34. doi:10.2147/IPRP.S162799.

18. Welch BE, Arif SA, Bloom TJ, et al. Report of the 2019-2020 AACP Student Affairs Standing Committee. Am J Pharm Educ. 2020;84(10):ajpe8198. doi:10.5688/ajpe8198.

19. Bureau of Labor Statistics, U.S. Department of Labor, Occupational Outlook Handbook, Pharmacists. https://www.bls.gov/ooh/healthcare/pharmacists.htm. Accessed June 17, 2021.

20. Pharmacists Top Most Trusted Professions in Gallup Poll. https://www.drugtopics.com/view/pharmacists-topmost-trusted-professions-gallup-poll. June 17, 2021. 